\title{
Correction to: Occupational exposure to organic solvents during pregnancy and childhood behavior: findings from the PELAGIE birth cohort (France, 2002-2013)
}

Nathalie Costet $^{1 *}$, Rémi Béranger ${ }^{2}$, Ronan Garlantézec ${ }^{2}$, Florence Rouget ${ }^{2}$, Christine Monfort ${ }^{1}$, Sylvaine Cordier ${ }^{1}$, Fabienne Pelé ${ }^{3}$ and Cécile Chevrier ${ }^{1}$

\section{Correction}

Following publication of the original article [1], the author noticed that a mistake occured during the final production step and asked to replace Table 2 with the correct version.

The correct version of the table has been included in this correction.

The original article has been corrected.

\begin{abstract}
Author details
${ }^{1}$ Epidemiological Research in Environment, Reproduction and Health, Univ Rennes, Inserm, EHESP, Irset-UMR_S 1085, 9, avenue du Prof. Léon Bernard, F-35000 Rennes, France. ${ }^{2}$ Univ Rennes, CHU Rennes, Inserm, EHESP, Irset-UMR_S, 1085 Rennes, France. ${ }^{3}$ Univ Rennes, CHU Rennes, Inserm, Irset-UMR_S 1085, CIC 1414, F-35000 Rennes, France.
\end{abstract}

Received: 27 August 2018 Accepted: 27 August 2018

Published online: 04 September 2018

\section{Reference}

1. Costet, et al. Occupational exposure to organic solvents during pregnancy and childhood behavior: findings from the PELAGIE birth cohort (France, 2002-2013), vol. 17; 2018. p. 63. https://doi.org/10.1186/s12940-018-0406-x

* Correspondence: nathalie.costet@univ-rennes1.fr

${ }^{1}$ Epidemiological Research in Environment, Reproduction and Health, Univ Rennes, Inserm, EHESP, Irset-UMR_S 1085, 9, avenue du Prof. Léon Bernard, F-35000 Rennes, France

Full list of author information is available at the end of the article

(c) The Author(s). 2018 Open Access This article is distributed under the terms of the Creative Commons Attribution 4.0 International License (http://creativecommons.org/licenses/by/4.0/), which permits unrestricted use, distribution, and reproduction in any medium, provided you give appropriate credit to the original author(s) and the source, provide a link to the Creative Commons license, and indicate if changes were made. The Creative Commons Public Domain Dedication waiver (http://creativecommons.org/publicdomain/zero/1.0/) applies to the data made available in this article, unless otherwise stated. 
Table 2 Behavior Subscales Observed at Age 2 and 6 (Observed Variables) and Loadings on Their Corresponding Latent Trait (SEM Crude Model, N=715, PELAGIE Cohort, France, 2002-2013)

\begin{tabular}{|c|c|c|c|c|c|}
\hline \multirow[t]{2}{*}{ Behavior subscales } & \multirow[t]{2}{*}{ Scale of origin ${ }^{a}$} & \multirow[t]{2}{*}{ \# items } & \multirow[t]{2}{*}{ Median scores (IQR) } & \multirow[t]{2}{*}{$\begin{array}{l}\text { Corresponding behavior } \\
\text { latent trait }\end{array}$} & \multirow{2}{*}{$\begin{array}{l}\text { Factor loading } \\
\lambda^{b}(95 \% \mathrm{Cl})\end{array}$} \\
\hline & & & & & \\
\hline \multicolumn{6}{|l|}{ At age 2} \\
\hline Emotional symptoms & $\mathrm{CBCL}$ & 3 & $2(0-2)$ & Internalizing & 1.00 \\
\hline Attention deficit/hyperactivity & $C B C L, P S B Q$ & 6 & $5(3-7)$ & Externalizing & $0.70(0.63,0.78)$ \\
\hline Aggression & $\mathrm{CBCL}$ & 3 & $1(0-3)$ & Externalizing & $0.44(0.36,0.53)$ \\
\hline Opposition & $\mathrm{CBCL}$ & 3 & $3(2-4)$ & Externalizing & $0.63(0.57,0.70)$ \\
\hline \multicolumn{6}{|l|}{ At age 6} \\
\hline Emotional symptoms & SDQ & 5 & $2(1-3)$ & Internalizing & $0.63(0.51,0.74)$ \\
\hline Peer relationship problems & SDQ & 5 & $1(0-2)$ & Internalizing & $0.52(0.42,0.62)$ \\
\hline Hyperactivity/inattention & SDQ & 5 & $3(1-4)$ & Externalizing & $0.61(0.53,0.69)$ \\
\hline Conduct problems & SDQ & 5 & $2(1-3)$ & Externalizing & $0.71(0.63,0.78)$ \\
\hline
\end{tabular}

Abbreviations: IQR, interquartile range $\left(25^{\text {th }}\right.$ percentile $-75^{\text {th }}$ percentile)

${ }^{a}$ CBCL: Child Behavior Checklist; PSBQ: Preschool Social Behavior Questionnaire; SDQ: Strengths and Difficulties Questionnaire

${ }^{\mathrm{b}}$ Standardized factor loading (95\% Confidence Interval) 